\title{
Are improvements in arterial stiffness associated with moderate physical activity and modulated by nitric oxide by-products in overweight adults?
}

\author{
T. M. Kearney ${ }^{1}$, M. H. Murphy ${ }^{2}$, G. W. Davison ${ }^{2}$, M. J. Okane ${ }^{3}$ and A. M. Gallagher ${ }^{1}$ \\ ${ }^{1}$ Northern Ireland Centre for Food and Health, University of Ulster, Coleraine BT52 1SA, ${ }^{2}$ Ulster Sports Academy, \\ University of Ulster, Newtownabbey, BT37 OQB and ${ }^{3}$ Dept of Clinical Chemistry, Altnagelvin Hospital, Western Health \\ and Social Care Trust, Londonderry BT47 6SB, UK
}

Arterial stiffness, measured via pulse wave velocity (PWV) has been shown to be a strong independent predictor of cardiovascular morbidity and all-cause mortality ${ }^{(1)}$. Healthy compliant brachial arteries normally have a PWV of $7-9 \mathrm{~ms}^{-1}$, whereas stiffer arteries tend to be in the range of $12-15 \mathrm{~ms}^{-1(2,3)}$. It has been hypothesised that regular physical activity improves endothelial function due to vascular shear stress causing an increased production of the vasodilator nitric oxide $(\mathrm{NO})^{(4)}$. We previously reported that six months of brisk walking was associated with a decrease in $\mathrm{PWV}^{(5)}$. Here we investigated whether the observed beneficial changes in PWV following regular brisk walking were associated with changes in serum nitrate and nitrite $\left(\mathrm{NO}_{\mathrm{x}}\right)$ (a surrogate marker for the vasodilator $\mathrm{NO}$ ) concentrations.

77 overweight sedentary individuals (19 males, 58 females; mean age 45.6 (sD 6.55) years; BMI 29.18 (sD 4.27$) \mathrm{kg} / \mathrm{m}^{2}$ ) participated in a randomised control trial and were allocated to one of three groups: control group (n25), walking with monthly telephone contact group (n25) and walking with weekly telephone contact group (n27). The walking groups were asked to incorporate $3 \times 10$ minute bouts of brisk walking into their daily routine on 5days/week and were contacted over six months on to provide support. The control group were given light stretching exercises to carry-out on 5days/week and were contacted on a monthly basis to control for attention effects. Percentage body fat (Tanita scales), BMI $\left(\mathrm{kg} / \mathrm{m}^{2}\right)$, and PWV were measured at baseline and repeated after six months with follow-up measurements taken four months later. PWV was measured using a sensor based device as described by McLaughlin et al. ${ }^{(2)}$. For the purposes of the present investigation, both walking groups were combined (n52) and telephone contact was included as a covariate. Time by group interactions were analysed using repeated measures two-way analysis of covariance (ANCOVA). Between subject differences were analysed using a one-way ANOVA with posteriori Tukey Honestly Significant Difference (HsD) test.

There was a significant decrease in PWV $(P<0.001)$ coupled with significant increases in $\mathrm{NO}_{\mathrm{x}}(P<0.001)$ over the 6 month intervention, in the walking group as compared to control, and these beneficial effects were sustained beyond the end of the intervention period (i.e. at 4 months follow-up) (Figure 1 and 2). Furthermore a strong negative correlation between $\mathrm{PWV}$ and $\mathrm{NO}_{\mathrm{x}}$ was also observed $(r=-0.65, P<0.001)$
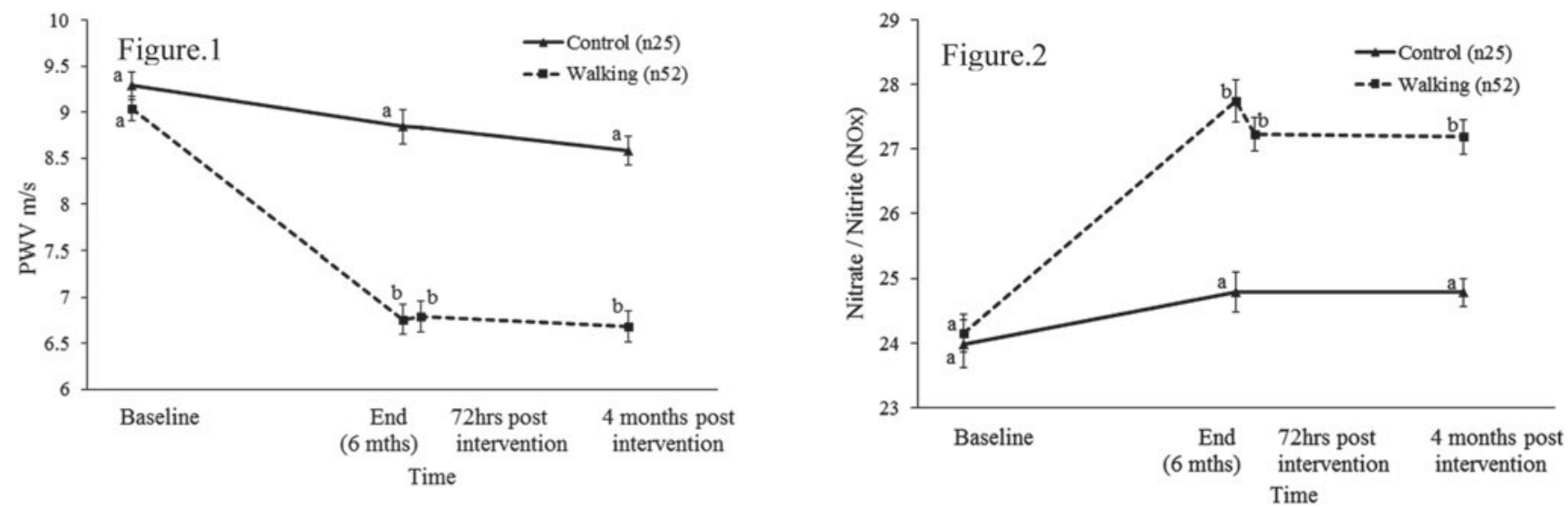

The results demonstrate that the beneficial changes in PWV observed with regular brisk walking for $3 \times 10$ minutes per day may be explained by concurrent increases in nitric oxide by-products in these overweight individuals.

1. Noor A et al. (2009) J Hypertens. 27, 2186-2191.

2. McLaughlin et al. (2003) Physiol Meas 24, 693-702.

3. Zambanini A et al. (2005) American J Physiol-Heart C 289(1): H270-H276.

4. Hambrecht R et al. (2000) New Engl J Med 342, 454-460.

5. Kearney TM et al. (2010) Proc Nutr Soc 69 (OCE5): E360. 